\title{
Reliability through Cooperative Work in Software Testing*
}

\author{
Tuomo Tuikka \\ Department of Information Processing Science, University of Oulu, \\ Linnanmaa, FIN-90570, Oulu, Finland, email:ttu@rieska.oulu.fi
}

\begin{abstract}
There are many papers pointing out ways to reach reliability in the product. Those papers are mostly dealing with the issue in quantitative manner. However, the reliability we in our everyday software production try to reach depends more or less on an agreement between people producing a product. This agreement is reached through cooperation during the process of software production. This is a paper to take a look at software testing and reliability with a view of cooperative work. Surely, all work is essentially cooperative work in that it depends upon others for its successful performance and software testing is no exception of this. Open question is what is the role of cooperative work in software testing and reliability of the product? The ideas presented here are examples based on the experience of the writer in the field of software testing. As a result cooperative work is seen to play essential role for reliability. Furthermore, a view of articulation work is suggested to be useful for further studies of cooperative work in software testing.
\end{abstract}

Keyword Codes: D.2.5; J.4; K.6.3;

Keywords: Software Engineering, Testing and Debugging; Social and Behavioral Sciences; Management of Computing and Information Systems, Software Management;

\section{SOFTWARE TESTING AND RELIABILITY}

Software testing is one part of the software production process and important phase so that reliable products can eventually be sent out to world market. As software producers we also have a responsibility of our products because people using them might lose their time and money due to errors in the program. Thus, we have goals for software testing such as high quality, user satisfaction, cost benefits.[1] Perhaps we could see software testing to be useful in two spheres like [1] does: "When carried out effectively in the commercial sphere, software testing will contribute to the delivery of higher quality software products, more satisfied users and lower maintenance costs. In the scientific sphere, good testing will result in more accurate and reliable results." Considering this division the goals of these two spheres could sometimes be contradictory. Questions like what is the level of results for (scientifical) testing or when is the product good enough for release arise (commercial pressure). Although software testing has a different significance to different people, usually quality of the product is we all aim at. Furthermore, quality of other kind, the quality of software process, certainly affects the ultimate quality, reliability, and safety of the software. While testing is no substitute for improving

\footnotetext{
*This work has been done in the Finnish part of the ESPRIT project 6225 COMIC, funded by F. C. T D. (Tekes). Author wishes to thank Tauno Tönning foundation for additional funding and Peter Carstensen and Carsten Sørensen of Risø National Laboratory in Roskilde, Denmark for comments and suggestions.
} 
the process, it does occupy crucial role in the overall software development process[2] and it is an integral part of any quality programme.[1] As [3] puts it: "Testing is one of the methods used by the Software Quality Assurance (SQA) group to ensure the quality of systems..." and [4] "The purpose of testing is to make quality visible". More accurately program testing could be defined as simply: "Testing is the process of executing a program with the intent of finding errors"[5]. Hetzel defines testing further by determining meeting requirements: "Testing is any activity aimed at evaluating an attribute or capability of a program or system and determining that it meets its required results" [4] There are several stages of testing including unit, integration, system, product, customer, and regression testing. These terms usually refer to the stage of the testing, not its form. This paper refers more to regression testing which is meant to assure that a new version of a system faithfully reproduces the desirable behaviour of the previous system [2]. Software testing techniques are discussed in [6, 7].

What we intuitively call "software reliability" is the probability of not encountering a sequence of inputs that leads to failure.[8] Reliability estimation, viewed by [2], attempts to predict the mean time to failure of software based on the experience of testing against an operational profile of the software use. Safety evaluation attempts to find and eliminate sources of failure and risk in software based on an understanding of the code, its requirements, and its environment of use.[2] Software reliability analysis, the estimation of the probability of software failure during a specified exposure period, as a subject of research is related to the wellestablished work on general system reliability theory. Many papers have been written on software reliability models. Overview e.g. [9]

Software is a difficult object to develop [8]: "Problems or fundamental limitations in software are: 1) Complexity, it takes time to develop, 2) Error sensitivity, no useful interpretation of tolerance is known for software, 3) Hard to test, software is notoriously difficult to test adequately. Moreover, it has been pointed out that testing carried out by selected test cases, no matter how carefully and well-planned, can provide nothing but anecdotes. 4) Correlated failures, in contrast to the situation with hardware systems, one cannot obtain higher reliability by duplication of software components. One simply duplicates the ertors. 5) Lack of professional standard."[2] Thus, besides difficult to develop, an important problem is whether test is adequate, that is, not only what test cases should be tested but also when testing should start and stop. We do have measures to lean on in trying to determine if our product is ready for release, but it is impossible to tell if our software is error free. Apparently there is a lot true of what is said about belief on a product trustworthiness: "We cannot test software for correctness, it is difficult to make accurate predictions of software reliability and availability, it is not practical to measure the trustworthiness of software. We consider a product to be trustworthy if we believe that the probability of it having a potentially catastrophic flaw is acceptably low."[8] There has been also critics on statistical methods for calculating the reliability of program. There is consensus among several researchers that there are some serious shortcomings of these models which need to be remedied before they can be used.[2] Many of the models rely on assumptions that are often not satisfied in practice. Another drawback of these models as [2] mentions is that they do not deal directly and thoroughly with the crucial question of when to stop testing the software. Well, if we cannot test software for correctness how do we get products out anyway? How do we agree on product reliability?

Objective of this paper is not to show problems of any models, but to present a view to cooperative work in software testing when reliability of the product is sought-after.Suggestion presented here is that agreement of reliability of software emerges through cooperative work in software production. Thus, e.g. required results (cf.[4]) of software are negotiable. To illustrate this argument I give an example of my personal experience in the field of software testing and especially in this case from work in regression testing. 


\section{COOPERATIVE WORK IN SOFTWARE TESTING}

The first place we find cooperation useful in software testing is the situation where we are trying to test our own product. Like [1] says:"We normally perceive our own products as correct. This means that, once we have made an error during the development phase, this becomes part of our product. Having made an erroneous assumption once, in reviewing or testing the product we are likely to make the same assumption again. To operate successfully, anyone testing their own software must be prepared to question every aspect of the product, ignoring any inclinations to assume the correctness of any previous work. For most of us this is difficult: our thought processes will follow the previously trodden path - to repeat the error"[1]. In case we work in a large software production environment, however, say for example 60 or more people, the role of software tester and programmer is usually divided. The nature of test situation determines then the nature of cooperation.[6]

All work is essentially cooperative work in a sense that it depends upon others for its successful performance (cf. [10]), and it is constituted by work processes that are related as to content, that is, processes pertaining to the production of a particular product or service. The definition for cooperative work used here is: "At the core of this conception of cooperative work is the notion of interdependence in work. In the sense that cooperative work occurs when multiple actors are required to do the work and therefore are mutually dependent in their work and must coordinate and integrate their individual activities to get the work done "[11]

Next example is a description how regression testing can happen. The problem studied is when software testing can be stopped and release sent out. Let's assume a software corporation has 65 people working on a product. That means we have many mutually interdependent actors in working on a product. In that group we have software developers, testing groups, and marketing people who are waiting for the delivery date to come. Figure 1 is simplified view where a circle means a position in a team.

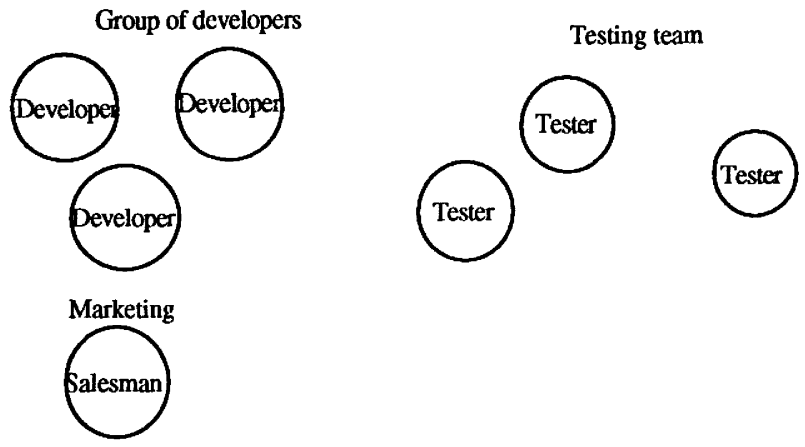

Figure 1. Simple description of software production group.

When we try to see 65 of them we get a little idea of the complexity we might be in when studying the organisation and work in it. In this simplified example we think that programmers and testers are those people who develop the product. Programmers are those who write the code, and testers test product, collect errors, and inform of them. However, if someone else finds an error, responsibles are notified. Every tester has his/her own area of responsibility, which is some part of the product. Product may consist of different parts which can be also products themselves. This part can be the user interface and its functions, compiler or a unit 
which can be thought to be tested as a whole. Usually everyone has several of these responsibility areas. Here we can see that many highly interconnected parts, components, concepts, lines of code created etc. are in the field of work. There are many actions for tester who tries to find errors in the program. Let's assume a tester finds a new error. This information must be told further, so that error can be repaired. Of course the founder of an error can be anyone in the company. At this stage the error goes for correction if it is thought to be worth correcting. Worthyness is examined by the group working on the development and programmer, because correction should be done in a reasonable time period and be relative to other work. When the error is corrected, information of it is given further, with data on how it should be tested. This way we ensure it will not happen again. (Figure 2)

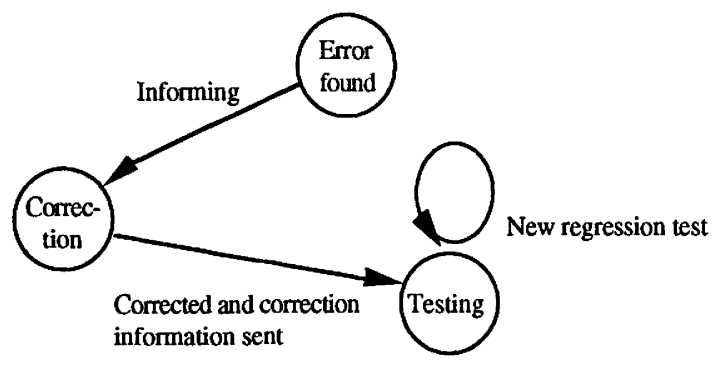

Figure 2. Simplified work process in case of finding an error.

New version of program should not include the error anymore. We need to be certain of that further on and a test operation is decided on it. This way we close the earlier problems of the product and also collect information of the product history. Test operation is decided by the test group leader usually, but with enough experience also tester is able to do it. Automatic test macro will be written for regression tests and finally the test operation is done automatically, unless the case is so difficult it needs special arrangements. When new version of the product is taken for tests, we can now ensure old error is fixed. This happens by automatic tests which are driven to new version. This phase is a mechanical procedure after automatic environment has been built. New tests can be added to the test set which can consist hundreds of tests. The result of testdrive should be that all tests pass, but sometimes you can find that old problem has come back again. Many broken errors might indicate a severe problem in product. For example, wrong file has been compiled during build up. One of the criterias for the maturity of the product could be error count and type of errors. Sometimes error type is needed to decide whether problem is important or not. In serious cases error must be fixed right away because leaving it would jeopardize the whole development process. Small problems could be cosmetic problems like wrong error messages etc. One generally interesting issue is where error has been. It might be possible to track the true cause of problems if we know where the bugs appear. This also could give a clue if the problem is very serious. With this knowledge it is possible to design further procedures and time it takes compare to other actions.

Previous description shows how work should go and usually goes. However, in final stages of product, date of release coming, everyone is doing their best to get all errors found and fixed. However, behind the curtains it is possible to find many problems. We have uncertainty of the real nature of an error, is the problem really how it looks like? There is also uncertainty how the test set is covering problem areas and what happens when error is fixed, does a fix create more and even worse problems. There is a constant change in utilisation of machines and equipment need to be renewed while technology develops. Learning and adaptation of new 
information is essential to get tests to right use environment. This is dynamism, and it is often caused from the fact that the work situations are characterised by handling a number of concepts, requirements, etc. that are dynamic by nature, i.e., events can happen at indeterminate times. This might result in change of the problem to be solved. Finally, supposing that we cannot test for correctness, how can we tell our product is ready. Can we trust the product enough for release? This is a question of negotiation. The marketing department may want to get a release despite of few minor bugs. But what is a minor bug? A minor bug may be a symptom for a far deeper problem. And a week or two more testing and fixing might hurt company stocks and investors.

In cooperative work people have to coordinate their work due to their mutual interdependency. Easiest way to see how this is done in our example is to look at how reliability is reached. Final reliability of product is a result of negotiations. There are several points that illustrate this in our example. First we need to inform responsible people of an error. Second, we have to do negotiations of worthiness of correction, the nature of error, probably about the results of tests and error severity in possible deliverable software. Issues for negotiation could be: What parameters of the product to discuss? What kind of errors could be acceptable in the product or worth correction? What new errors you could get from correcting old ones? Are we going to change anything we have earlier accepted as a standards? Through this process we finally reach an agreement (or not) that our product is good for release. Much information can be attained from formal reliability models, but they are only an aid for software testing work. Thus, the decision of release is made in meetings, discussions and negotiations and we can argue that cooperative work plays important role in software testing.

Furthermore, in a large software production company we can see that, besides mutually interdependent actors, many highly interconnected parts, uncertainty and dynamism characterise work environment and work. By Woods (1988) [12] they characterise the dimensions of complexity. Complex work situations are most often handled in a distributed cooperative work arrangement with mutually interdependent actors. In this case all activities are heavily intertwined and a decomposition into small isolated test activities is impossible [13], this means that division of labor in software testing is not only a matter of structuring the program properly[14]. Thus, many actors and perspectives are involved. This, in turn, requires articulation work to be performed by the people involved.[15] In the words of Strauss [16], articulation work is "a kind of supra-type work in any division of labor, done by various actors. Articulation work amounts to the following. First, the meshing of the often numerous tasks, clusters of tasks, and segments of the total arc. Second, the meshing of efforts of various workers (individuals, departments, etc.). Third, the meshing of actors with their various types of work and implicated tasks." For articulation purposes, activities such as allocating resources, planning and scheduling tasks, monitoring the state of affairs in the development and test process, classifying and prioritizing, distributing information, and negotiating requirements, priorities, etc. are essential.[15] Consequently the agreement of software maturity is reached through articulation work and reliability is more like common understanding on state of software and belief that software works on certain conditions.

\section{DISCUSSION}

A cooperative ensemble of people in software production and in testing process must find an agreement that the product is reliable enough for release. Still, disagreement can be possible even though the product has been released and market forces decide the success of the product. More studies of cooperative work and its computer support can be found in reseach field of Computer Supported Cooperative Work [17-19]. However, nature of software testing and articulation work are seldom addressed directly in the literature although cooperative work affects deeply into the quality of our products. Further studies of cooperative work would be useful for developing tools to support cooperative work in software testing which is one goal of my research. 


\section{REFERENCES}

1. Parrington N. and Roper M., Understanding Software Testing. 1989, Chichester, England: Ellis Horwood Limited.

2. Dalal S. R., Hogan J. R. and Kettering J. R.. Reliable Software and Communication: Software Quality, Reliability, and Safety. in 15th International Conference on Software Engineering. 1993. Baltimore, Maryland, USA: IEEE Computer Society Press, Los Alamitos, California, USA. p. 425-435.

3. Yourdon E., Software Quality Assurance in the 1990s. in Sixth Annual Pacific Northwest Software Quality Conference. 1988. Portland, Oregon, USA: p. 3-33.

4. Hetzel B., The Complete Guide to Software Testing. 1988, Wellesley, MA, USA: QED Information Sciences Inc. 284.

5. Myers G.J., The Art of Software Testing. 1979, New York: John Wiley and Sons.

6. Beizer B., Software Testing Techniques. Second Edition ed. 1990, New York: Van Nostrand Reinhold.

7. Beizer B., Software System Testing and Quality Assurance. 1984, New York: Van Nostrand Reinhold Company Inc. 358.

8. Parnas D. L., Schouwen A. J. v. and Kwan S. P., Evaluation of Safety-Critical Software. Communications of the ACM, 1990. 33(6): p. 636-648.

9. Malaiya Y. K. and Srimani P. K., Software Reliability Models: Theoretical Developments, Evaluation and Applications. 1990, Los Alamitos, California, USA: IEEE Computer Society Press.

10. Bannon L. and Schmidt K., CSCW: Four Characters in Search of a Context, in Studies in Computer Supported Cooperative Work. Theory, Practice and Design, J. M. Bowers and S. D. Benford, Editors. 1991, North-Holland: Amsterdam etc. p. 3-16.

11. Simone C.and Schmidt K., Computational Mechanisms of Interaction for CSCW1993, COMIC, ESPRIT Basic Research Project 6225: Lancaster, United Kingdom.

12. Woods D. D., Coping with complexity: the psychology of human behavior in complex systems, in Tasks, Errors and Mental Models. A Festschrift to celebrate the 60th birthday of Professor Jens Rasmussen, L. P. Goodstein, H. B. Andersen and S. E. Olsen, Editors. 1988, Taylor \& Francis: London etc. p. 128-148.

13. Petchenik N. H., Practical Priorities in System Testing. IEEE Software, 1985. 2(5): p. 18-23.

14. Parnas D. L., Software Aspects of Strategic Defense Systems. Communications of the $A C M, 1985$. 28(12): p. 1326-1335.

15. Carstensen P. H., Tuikka T. and Sørensen C., "Are we done now?" Towards Requirements for Computer Supported Cooperative Software Testing. in Precedings of the 17th Information systems Research seminar In Scandinavia (IRIS 17). 1994. IsoSyöte Conference Centre, Finland: University of Oulu, Department of Inf. Processing Science, Finland. p. 424-438.

16. Strauss A., Work and the Division of Labor. The Sociological Quarterly, 1985. 26(1): p. 1-19.

17. Schmidt K., Simone C., Carstensen P., Hewitt B. and Sørensen C., Computational Mechanisms of Interaction: Notations and Facilities, in Computational Mechanisms of Interaction for CSCW, C. Simone and K. Schmidt, Editors. 1993, Esprit BRA 6225 COMIC: Lancaster, England. p. 109-164.

18. Greif I., ed. Computer-Supported Cooperative Work:A Book of Readings. 1988, Morgan Kaufmann Publishers Inc.: San Mateo, USA.

19. Michelis G. D., Simone C. and Schmidt K., Proceedings of the Third European Conference on Computer-Supported Cooperative Work. 1993. Milan, Italy: Kluwer Academic Publishers. .[1] N. Parrington and M. Roper, Understanding Software Testing. 1989, Chichester, England: Ellis Horwood Limited. 